\title{
Influence of packing density and fillers on thermal conductivity of polymer powders for additive manufacturing
}

\author{
Francesco Sillani $^{1,2}$ (D) $\cdot$ Fabian de Gasparo ${ }^{2} \cdot$ Manfred Schmid $^{1} \cdot$ Konrad Wegener $^{2}$
}

Received: 4 January 2021 / Accepted: 15 April 2021 / Published online: 10 May 2021

(C) The Author(s) 2021

\begin{abstract}
Additive manufacturing of polymer powders is nowadays an industrial production technology. Complex thermal phenomena occur during processing, mainly related to the interaction dynamics among laser, powder, and heating system, and also to the subsequent cool-down phase from the melt to the parts. Thermal conductivity of the powder is a key property for material processing, since an inhomogeneous temperature field in the powder cake leads to uneven part properties and can strongly limit productivity because only a smaller portion of the build chamber can be used. Nevertheless, little is known about the relationship between thermal conductivity, packing density, and presence of fillers, which are used to enhance specific properties such as high temperature resistance or stiffness. The development and consequent validation of a device capable of measuring thermal conductivity as a function of powder packing density are then extremely important, providing an additional tool to characterize powders during the development process of new materials for PBF of polymers. The results showed a positive correlation between packing density and thermal conductivity for some commercially available materials, with an increase of the latter of about 10 to $40 \%$ with an increase of the packing density from 0 to $100 \%$. Problems arose in trying to replicate the compaction state of the powder, since the same amount of taps led to a different packing density, but this is a known problem of measuring free-flowing powders such as the ones used for additive manufacturing. Regarding fillers, an increase of about 40 to $70 \%$ of thermal conductivity when inorganic fillers such as carbon fibers are added to the neat polymer was observed, and the expected behavior following the rule of mixture was only partially observed.
\end{abstract}

Keywords Thermal conductivity - Powder - Polymer - Powder bed fusion - Selective laser sintering - Duraform PA12 . Transient hot wire $\cdot$ Duraform HST $\cdot$ iCoPP $\cdot$ Composite $\cdot$ Compaction behavior $\cdot$ SmarTap

\section{Introduction}

Additive manufacturing (AM) is a family of manufacturing technologies for which an increasing interest is arising in different industries. Metals, polymers, and even ceramic materials can be nowadays processed using AM [1]. AM technologies can be categorized depending on the feedstock that is being processed and, according to ISO/ASTM 52900 standard [2], three main families exist: liquids, filaments/wires, and powders.

Francesco Sillani

sillani@inspire.ethz.ch

1 Inspire, Innovation Center for Additive Manufacturing Switzerland (icams), Fürsterlandstrasse 122, 9014 St. Gallen, Switzerland

2 ETH Zürich, Institute of Machine Tools and Manufacturing (IWF), 8092 Zürich, Leonhardstrasse 21, Zürich, Switzerland
Powder bed fusion (PBF) comprises most of AM with powder feedstock, and depending on the heating medium (lasers, IR lamps, etc.) and material (metal, polymer, or ceramic), it is named in different ways, the most common ones being laser sintering (LS, laser as heating medium, polymer as feedstock - official nomenclature PBF-LB/P) and selective laser melting (SLM, laser as power source, metal as feedstock-official nomenclature PBF-LB/M) [1, 2].

LS can be sketched as a two-step process: recoating and laser-induced melting. First, powder is applied in layers with predefined thickness (typically $100 \mu \mathrm{m}$ ) while preheated above the crystallization temperature and below the melting point of the polymer, in the so-called sintering window [3]. Then, a laser beam scans the cross-section of the part on the build platform in order to selectively melt the polymer. This process is repeated for hundreds or thousands of layers, until the part is finished.

The feedstocks used are typically optimized in many ways, the most important one being their rheological 
behavior, described as flowability. The term "flowability" is intuitively used to describe the ease of flow of a powder but is not a physical quantity, and a good flowability is especially important in PBF, since it allows creating thin, homogeneous, and dense layers, an important pre-requisite for obtaining good parts. Flowability can be measured using different methodologies, such as angle of repose [4] or powder rheometers [5], and the most relevant ones for PBF are reviewed by [6], who mentions that the testing device should be as close as possible to the process conditions. In fact, [7] reports that powder flow in industrial processes can be hardly assessed in an universal way and different expressions of flowability can refer to the same powder flow. In the case of PBF, the powder flow is actually multi-phase, with several key steps (recoating, compaction in the feeders, compaction in the build chamber, etc.), which makes extremely challenging the choice of the best measurement technique.

One of the many powder flows involved is the compression flow, which can be exerted onto a powder in order to determine its ability to compact. The ASTM B417 [8] standard suggests a standardized methodology to determine the so-called bulk density $\rho_{B}$, which is the density of the powder in the unconfined state. On the other hand, the ASTM B527 [9] standard prescribes a methodology to ascertain the powder tap density $\rho_{t}$, and consists in letting it compact under its own weight upon tapping, which is a vertical displacement of $3 \mathrm{~mm}$ followed by free fall, for 3000 times. Recently, [10] reported a novel methodology to characterize the compaction behavior of AM feedstock using a commercially available tapping device modified with a camera that can automatically extrapolate the density of the powder at each tap, as shown in Fig. 1.

Another set of properties which is extremely important for the successful completion of the process is related to the thermal behavior of the material: enthalpy of fusion and supercooling window, also called sintering window, are extremely important properties for a suitable choice of process parameters in LS [3]. These are inherent properties of the material that can be easily measured with a differential scanning calorimetry (DSC).

Thermal conductivity is another key property for PBFLB/P, since it affects different steps of the process: during recoating, the colder powder should reach as fast as possible the temperature of the powder bed (Fig. 2a), limiting the creation of crystallization-induced stresses in the previously molten layer and also reaching the correct temperature for laser processing. After the laser beam has passed, the part is slowly cooled down to the temperature of the powder cake (Fig. 2b) until thermal equilibrium is reached (Fig. 2c). At the same time, the entire powder cake cools down to room temperature. a)
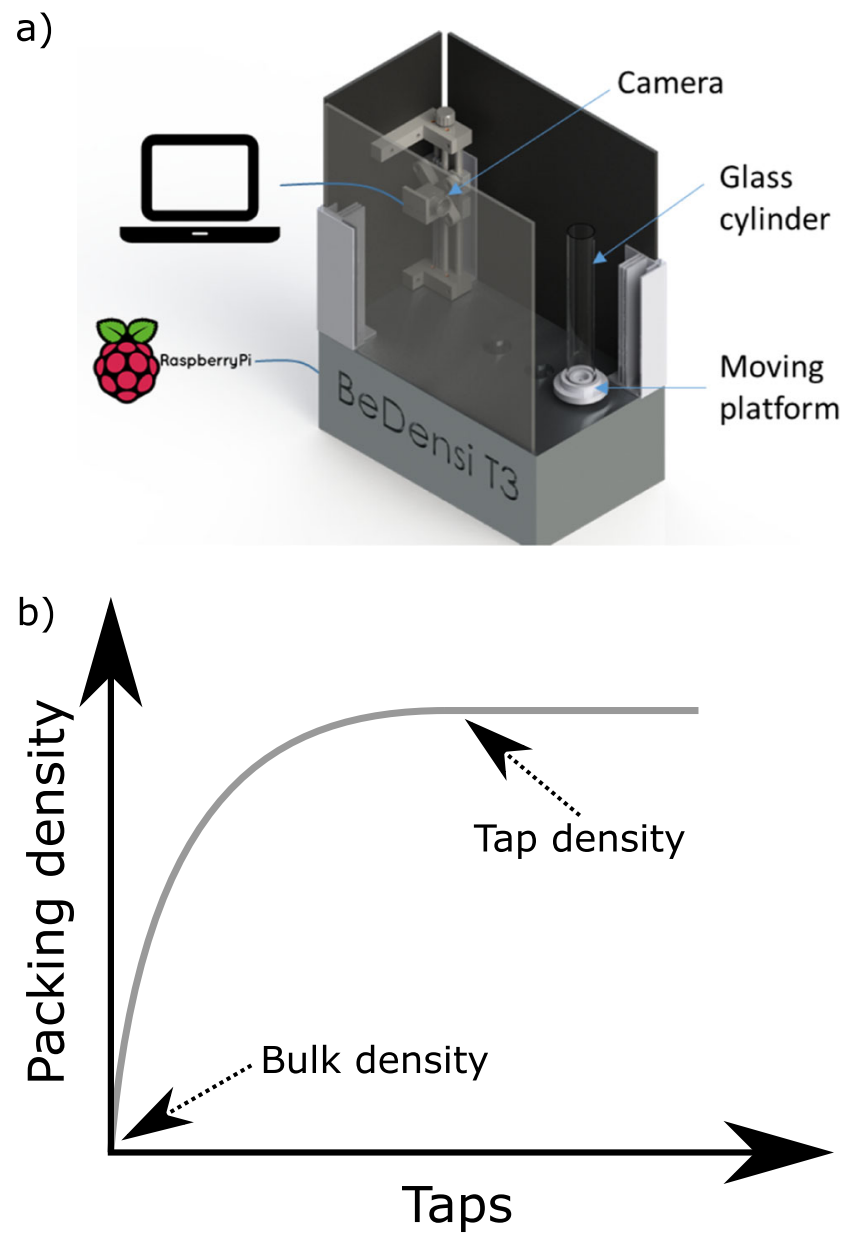

Fig. 1 a SmarTap device [10]. b Typical compaction curve with packing density plotted against tap count

Thermal conductivity is reported to be dependent on the compaction state of the powder: [11] investigated this relationship in the bulk and tap density states for commercially available PA12, the most common polymer powder used for PBF-LB/P [1].

Nevertheless, this quantity is not typically studied nor reported for commercial powders suitable for powder bed fusion, but knowledge about it is extremely important for modeling and understanding the process. The scope of the current work is thus to introduce a new tool for measuring in a semi-automated manner the thermal conductivity of nonconductive powders based on the well-known analytical solution of transient hot wire probe. Such a measuring equipment is designed to be mounted onto a modified tapping machine, for which more details are available elsewhere [10], and allows then to measure the thermal conductivity as a function of the number of taps. Therefore, in this study, the relationship between thermal conductivity and packing density will be explored. Moreover, since interest over composite materials has been growing for years [1], also the influence of fillers on thermal conductivity 

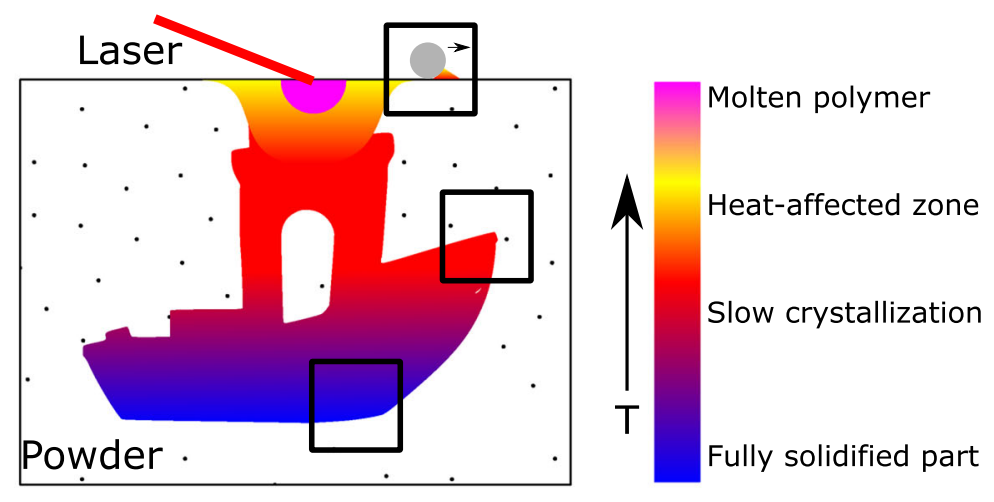

a)

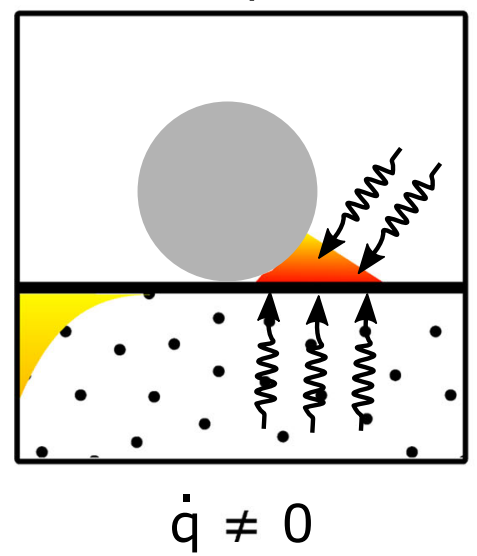

b)

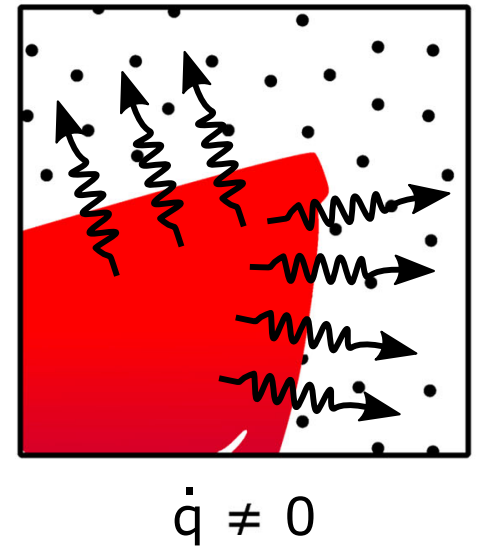

c)

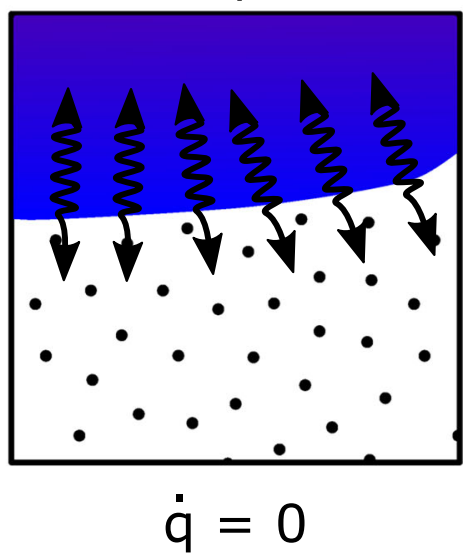

Fig. 2 Powder-related heat transfers in SLS, $\dot{q}$ is the time-dependent heat flow a $\dot{q}$ from the powder bed heater and powder bed to the cold powder coming from the feeding system; $\mathbf{b} \dot{q}$ from the part to the surrounding powder cake; $\mathbf{c} \dot{q}=0$ when the solidified part reaches the temperature of the powder bed

of commercially available polymer powders for PBF-LB/P will be studied.

\section{Materials and methods}

\subsection{Powder}

The materials used in this study are summarized in Table 1. All of them are commercial PBF-LB/P grades except for iCoPP-CF, which is a research grade, dry blend of iCoPP and short carbon fibers.
The use of the rule of mixture to estimate thermal conductivity is not reflecting the necessity of achieving a percolation threshold in order to allow an enhancement of this property. Nevertheless, it represents a first approximation to start predicting the possible values regarding the powder behavior.

\subsection{Transient hot wire}

The transient hot wire method is a well-known and long-term established measurement technique for thermal conductivity of fluids and solids, dating back to the early

Table 1 Materials used in this study, with filler content in volume percentage and thermal conductivity for the solid materials from literature (base polymers) and from rule of mixture (composites)

\begin{tabular}{|c|c|c|c|c|}
\hline Commercial name, short name & Producer & Base polymer & Filler & Thermal conductivity $\left(\mathrm{mWm}^{-1} \mathrm{~K}^{-1}\right)$ \\
\hline Duraform PA12 (PA12) & 3D Systems (Rock Hill, SC, USA) & Polyamide 12 & - & 200 \\
\hline Duraform HST (PA12-MF) & 3D Systems (Rock Hill, SC, USA) & Polyamide 12 & $11 \%$ wollastonite & 460 \\
\hline iCoPP (iCoPP) & inspire irpd (St. Gallen, Switzerland) & Polypropylene & - & 200 \\
\hline iCoPP (iCoPP-CF) & inspire irpd (St. Gallen, Switzerland) & Polypropylene & $10 \%$ carbon fibers & 760 \\
\hline
\end{tabular}


nineteenth century [12]. It is based on the analytical solution of the idealized case of an infinitely long linear heat source, and more specifically on the temperature transient occurring when power is applied with a step function [13, 14]. The heating of the wire through Joule heating brings also an increase in electrical resistivity, which can be easily measured and converted into temperature: this way, the wire itself can act both as heating medium and thermometer, allowing to gauge the transient of the temperature depicted in Fig. 3.

The thermal conductivity can then be obtained by using the analytical solution of Eq. 1 and assuming perfect temperature equilibrium (hence infinite heat transfer coefficient) between the linear heat source and the surrounding heat conducting medium for any time $t$ :

$\frac{\partial T}{\partial t}=\kappa \nabla^{2} T$

where $T$ is the temperature field, $t$ the time, and $\kappa$ the thermal diffusivity defined as:

$\kappa=\frac{\lambda}{\rho \cdot c_{P}}$

where $\lambda$ is the thermal conductivity, $\rho$ the density, and $c_{P}$ the specific heat capacity at constant pressure.

The start and boundary conditions for solving (1) for an infinitely long, cylinder-shaped heat source in polar coordinates were reported already by [14]:

$\begin{cases}\Delta T(r, t)=0 & \text { for any } t \leq 0 \text { and any } r \\ \lim _{r \rightarrow 0} r \frac{\partial T}{\partial r}=-\frac{q}{2 \pi k} & \text { for any } t \geq 0 \\ \lim _{r \rightarrow \infty} \Delta T(r, t)=0 & \text { for any } \geq\end{cases}$

where $r$ is the radial coordinate in a cylindrical coordinate system centered co-axially to the heat source.

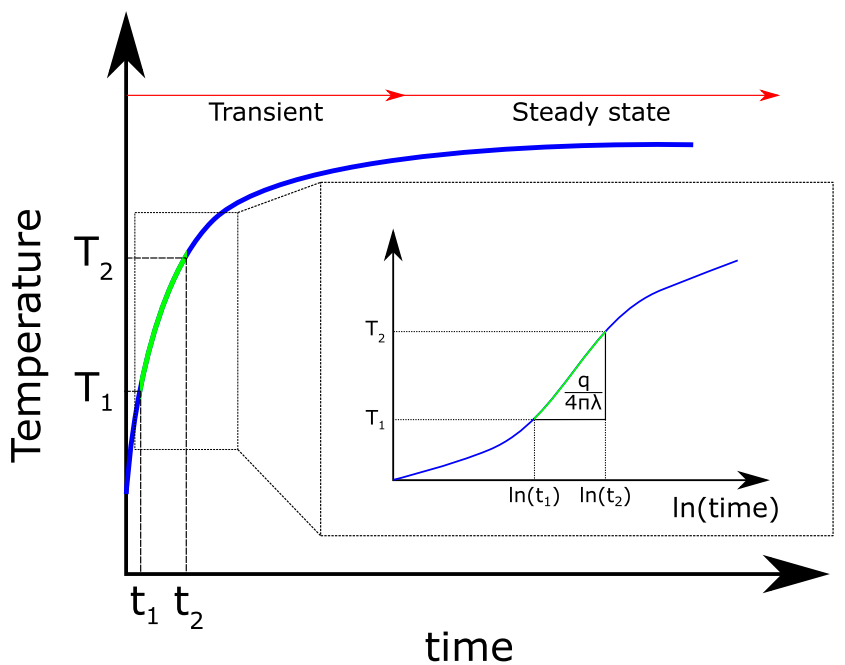

Fig. 3 Temperature evolution in the linear heat source upon application of a step function for the electric power
The temperature increase $\Delta T$ at the linear heat source with radius $a$ is then given as:

$\Delta T(a, t)=\frac{q}{4 \pi \lambda} \ln \frac{4 \kappa t}{a^{2} \exp e}$

This solution describes indeed the radial heat loss of an infinite line source of constant flux per unit length $q$ applied step-wise at $t=0$ through conduction into an infinite medium of constant thermal diffusivity $\kappa$.

Then, the thermal conductivity $\lambda$ can be obtained from knowledge of $\Delta T$ as a function of time by calculating the slope of the linear portion of temperature rise vs. the logarithm of the time as shown in Fig. 3:

$\lambda=\frac{q}{4 \pi \Delta T} \ln \Delta t$

\subsection{Thermal conductivity measuring setup}

A measuring apparatus is built according to the theory illustrated in Section 2.2 and similarly to what is reported by [15].

As shown in Fig. 4, a thin platinum wire (MaTecK $\mathrm{GmbH}$, Jülich, Germany) with diameter $25 \mu \mathrm{m}$ was soldered onto a AWG 30 copper wire (in black), which was connected to a constant voltage source through a transistor and to a measuring device, both controlled by an Arduino Mega as shown in Fig. 4.

At $t=0 \mathrm{~ms}$, a current $I$ is generated with a step function. The measurement device is used to determine $I$ and $V$ simultaneously at a sampling frequency of $200 \mathrm{~Hz}$, allowing the calculation of the resistance of the wire $R$ as a function

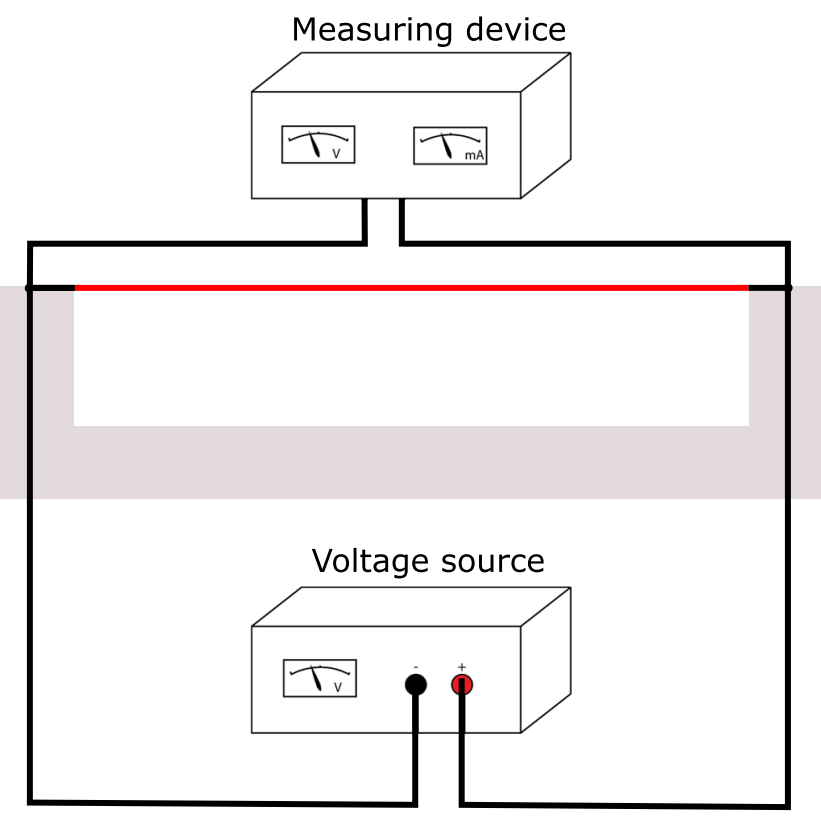

Fig. 4 Measuring setup with the Pt wire in red 
of the time $t$ according to Ohm's law. The resistance of the wire can be easily converted into a temperature through the temperature coefficient of resistivity $\beta$, which for Pt is equal to $3.729 \times 10^{-3} \mathrm{~K}^{-1}$ [16].

Data is transferred to Matlab, where all the necessary computations are performed in order to calculate the thermal conductivity of the sample. The voltage $V$ supplied by the generator is selected in order to minimize the temperature increase of the wire while providing a good signal-tonoise ratio. This is especially crucial for polymeric samples, where the $\Delta T$ induced by the measurement should not exceed $10^{\circ} \mathrm{C}$ in order to avoid sticking of the powder on the wire. Therefore, $10 \mathrm{~min}$ of cooling time between one measurement and the next in order to allow dissipation of the excess heat was planned.

\subsection{Integration with the tapping setup}

In order to perform measurements of the thermal conductivity as a function of the tapping density, the apparatus introduced in Section 2.3 is installed in a specifically designed holder. Figure 5 depicts the setup with the two cylinders, one on top of the other, while the blue element is the wire holder.

Since compaction upon tapping only results in a limited decrease of the height of the powder, a transparent top cylinder made of PETG is put on top of the transient hot wire cylindrical holder in order to allow the camera to detect the powder height as a function of taps [10].

\section{Results and discussion}

The data analysis is carried out according to the workflow shown in Fig. 6:

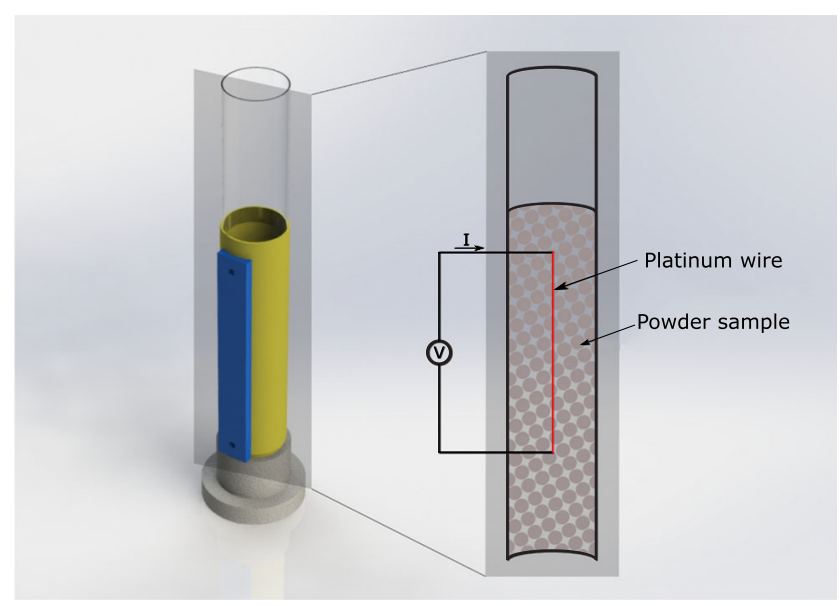

Fig. 5 Powder holder for the SmarTap
The resistance of the wire is measured (a), and converted into a temperature difference upon knowledge of the temperature coefficient of resistivity $\beta$ (b). Then, the thermal conductivity $\lambda$ for that specific measurement can be calculated, and finally plotted against the packing density of the powder at that specific tap count (c).

\subsection{Validation and repeatability}

In order to allow sufficient time for cool-down (see Section 2.3), the thermal conductivity was measured only at six different tap counts: 0 (upon filling, reference), 10, 50, 100, 500, and 1000. Also, for an initial validation, three measurements with PA12 were conducted at each tap count to gauge the sensitivity of the thermal conductivity measurement. Results are reported in Fig. 7.

As can be seen, the standard deviation of the measurement is quite good, with an overall average $\overline{S D}$ of about $3 \%$ across all packing densities $i$ :

$\overline{S D}=\frac{1}{6} \cdot \sum_{i=1}^{6} S D_{i}$

and a maximum of about $5 \%$.

The thermal conductivity of PA12 is in good agreement to what was reported by [11], although the transient hot wire method used in this work is very different from the methodology used by the other authors.

\subsection{Influence of packing density on thermal conductivity}

The results of the thermal conductivity measurements at different packing densities, are presented in Fig. 8.

It can be observed that error bars occur in both $\mathrm{X}$ and $\mathrm{Y}$ directions. This is due to the fact that the packing density is obtained upon application of a certain number of taps starting from an unknown compaction state, which is originated from filling in the sample holder. Therefore, an error bar in the $\mathrm{X}$ direction is needed to take into account this uncertainty. Regarding the error bars along Y, the measurements reported in Fig. 7 confirm that the thermal conductivity values hereby obtained are precise. These error bars originate then from the difficulty of replicating the exact compaction state when applying a certain amount of taps, a typical problem when dealing with free-flowing powders. A possible solution to overcome this problem would be to determine the packing density in real-time during the experiment, in order to carry out the thermal conductivity measurement always at the same packing density value.

In order to compare materials with different absolute packing densities, a normalization on the first and last data 
a)

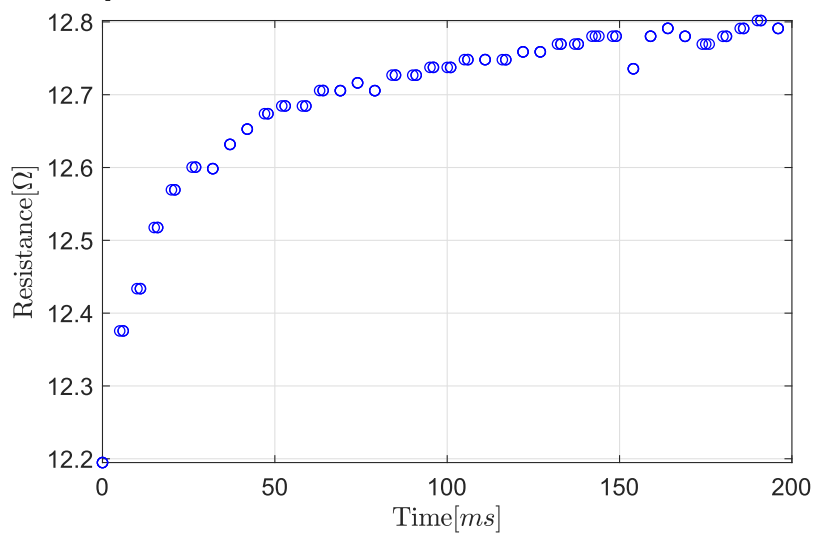

b)

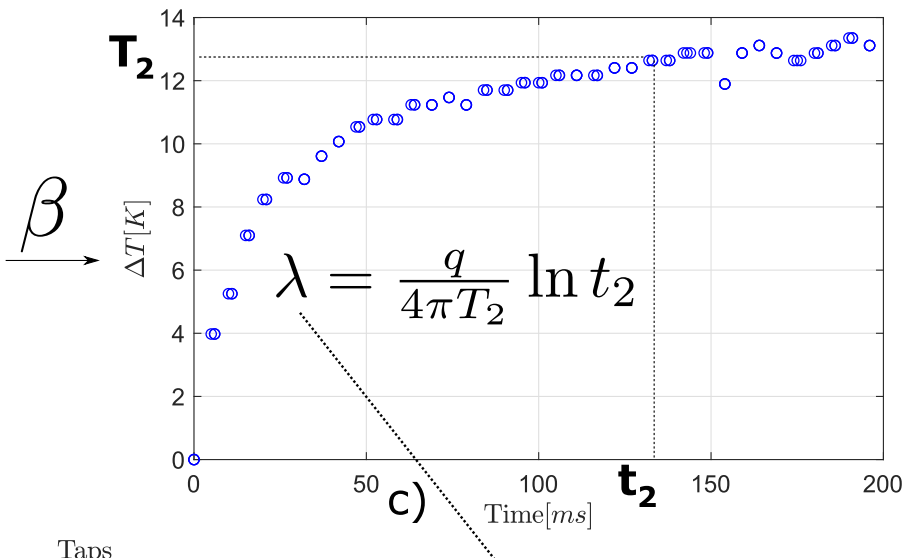

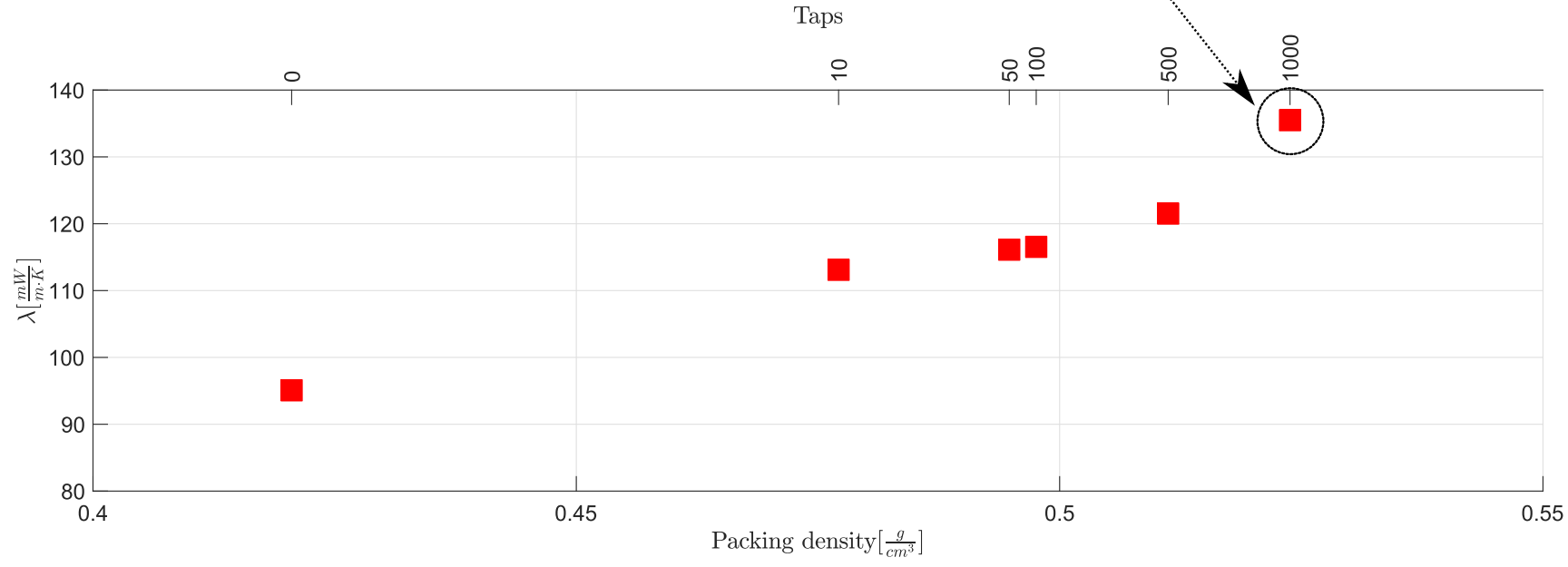

Fig. 6 Data analysis for every measurement. Resistance is determined during the transient $\mathbf{a}$ and converted into a temperature difference $\mathbf{b}$, which is then used to calculate $\lambda \mathbf{c}$

points of the packing density has been carried out, similarly to [10], according to:

$\tilde{\rho}_{i}=\frac{\rho_{i}-\min \left(\rho_{i}, \forall i\right)}{\max \left(\rho_{i}, \forall i\right)-\min \left(\rho_{i}, \forall i\right)} \cdot 100$

with $i$ being the tap count, ranging from 0 to 1000 .

The resulting curves for the neat polymers are reported in Fig. 9.

Polyamide 12 and polypropylene have very similar thermal conductivities in the solid phase, both around $200 \mathrm{mWm}^{-1} \mathrm{~K}^{-1}$ [17]. Therefore, the absolute difference between the two polymers at low packing densities is due to the different compaction state, caused by inherent powder properties such as shape and size distributions. In [18] is reported already an excellent flowability for iCoPP, being this powder characterized by an almost perfect spherical shape due to its production process through melt emulsification. On the other hand, [19] reported a shape distribution for PA12 with more elongated particles ("potato" shape), and thus worse flowability, with similar particle size distribution (PSD) compared to iCoPP.
For iCoPP, no significant difference arises in thermal conductivity when plotted against packing density, since 10 taps were enough to turn from 0 to more than $70 \%$ of the packing density. On the contrary, PA12 shows an expected linear increase in thermal conductivity upon increase of the packing density, with a variation from 90 to $130 \mathrm{mWm}^{-1} \mathrm{~K}^{-1}$.

\subsection{Influence of fillers on thermal conductivity}

Development and usage of composite materials and blends in PBF is growing, since fillers allow enhancing or even adding new properties to the matrix materials. In this particular case, it was decided to focus on the influence of fillers which act as a mechanical reinforcement to the base polymer. Duraform HST is a commercially available feedstock based on PA12 reinforced with wollastonite fibers, a ceramic material that drastically improves the heat deflection temperature [20]. The thermal conductivity of solid wollastonite is reported to be $2700 \mathrm{mWm}^{-1} \mathrm{~K}^{-1}$ [21], about thirty times the one of PA12 Fig. 10. 
Fig. 7 Thermal conductivity vs. packing density for PA12, three measurements per tap count equal to $0,10,50,100,500$, and 1000

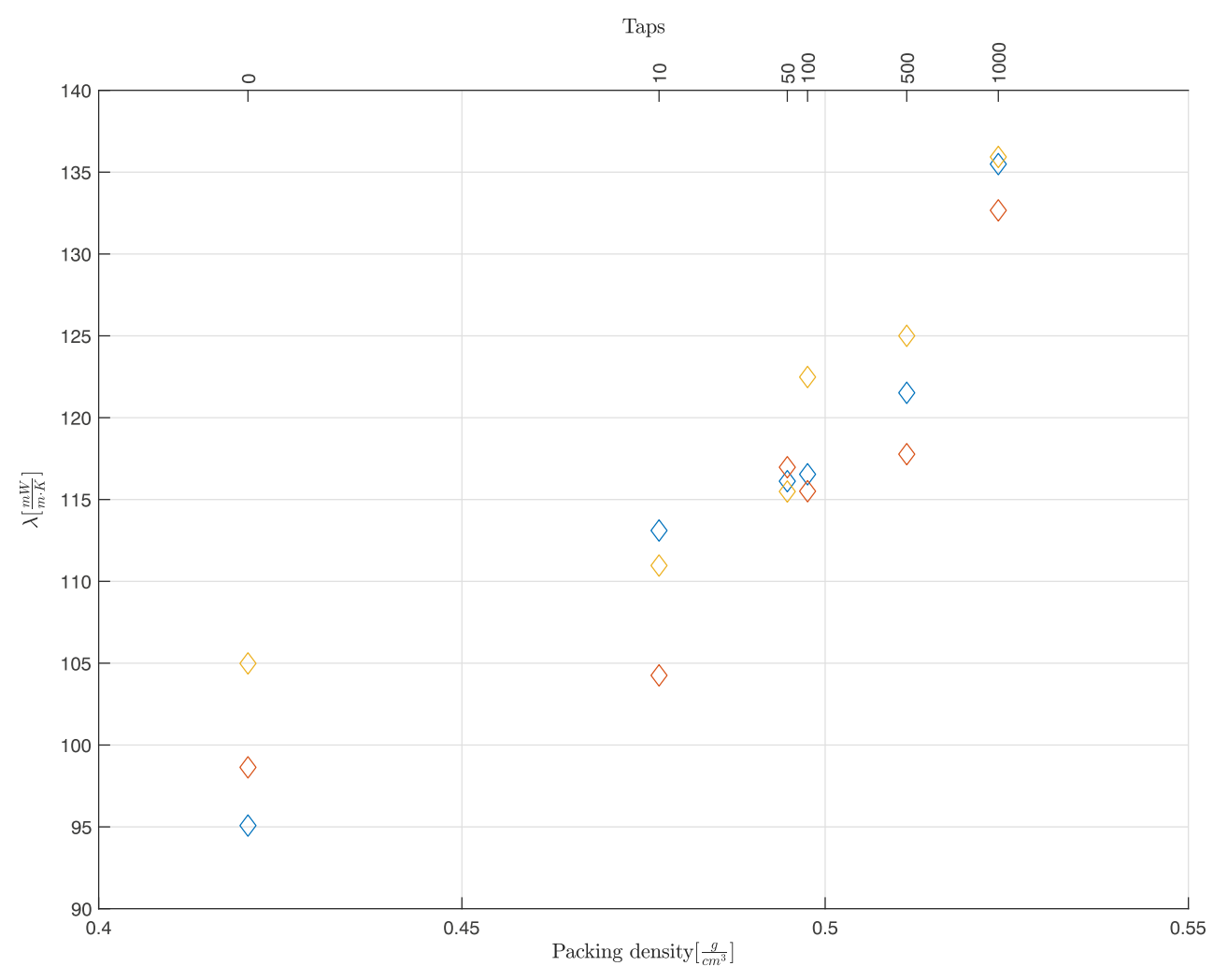

Thermal conductivity of PA12 is drastically enhanced thanks to wollastonite, with an increase of $60 \mathrm{mWm}^{-1} \mathrm{~K}^{-1}$ to $80 \mathrm{mWm}^{-1} \mathrm{~K}^{-1}$ depending on the compaction state. The increase was expected, but it is not as big as the rule of mixture would have predicted (around $350 \mathrm{mWm}^{-1} \mathrm{~K}^{-1}$ ). This is a clear indication that the processing conditions (laser power, energy density, etc.) need to be adapted in order to compensate for the different thermal conductivity of the powder, for example higher preheating temperature in the feeder. The same linear trend with packing density is observed also in PA12-MF, and again both powders show a

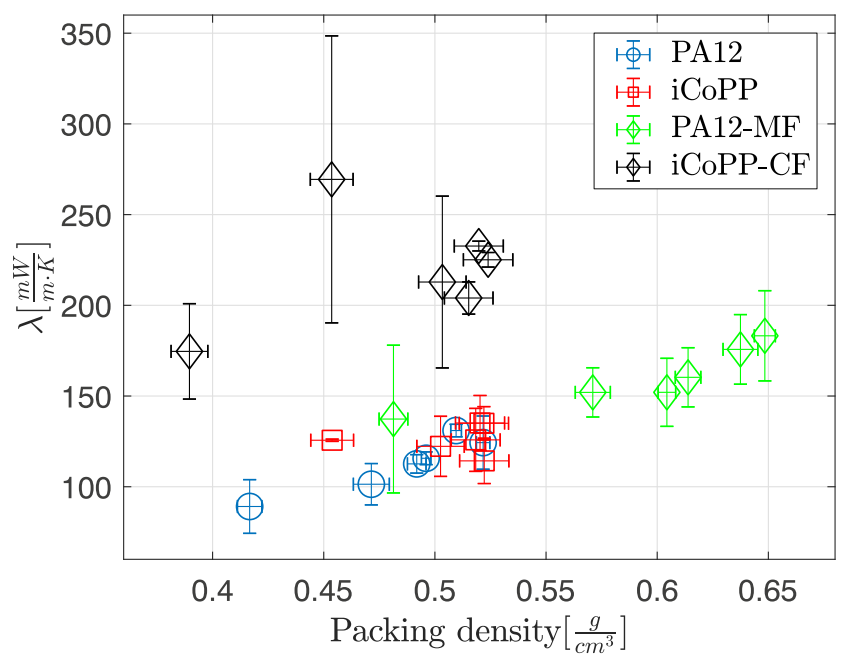

Fig. 8 Thermal conductivity vs. packing density for all materials very good flowability since they reach more than $50 \%$ of the tap density in only 10 taps.

iCoPP-CF is a research grade composite developed at inspire, based on a polyolefine available as production material for many years [18]. This composite material is obtained through dry-mixing of carbon fibers and neat $\mathrm{iCoPP}$ in order to enhance elastic modulus and tensile strength, while maintaining the typical properties of a polypropylene (chemical resistance, low melting temperature, etc.). Carbon fibers are known to have different

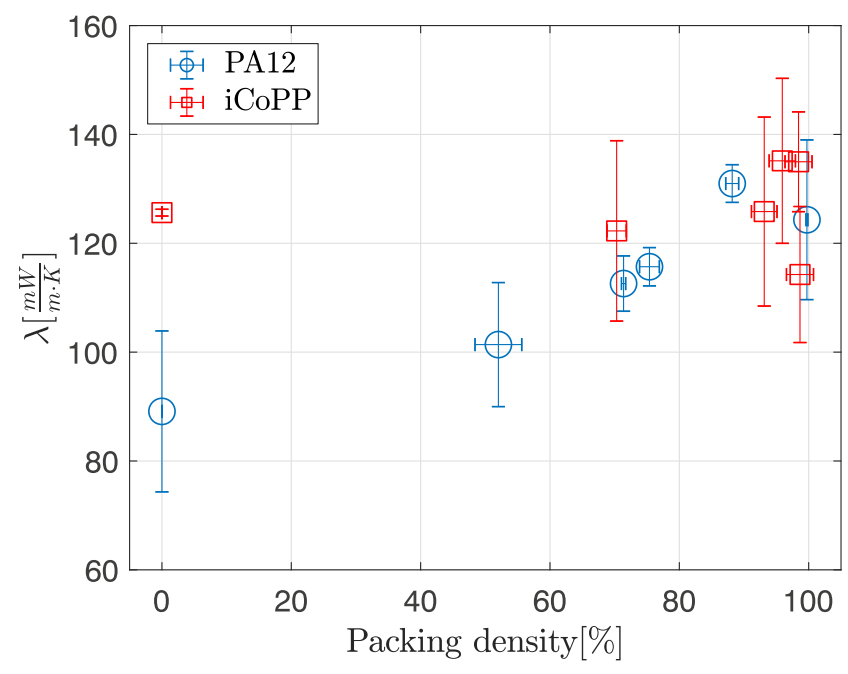

Fig. 9 Thermal conductivity vs. normalized packing density for the neat polymers 


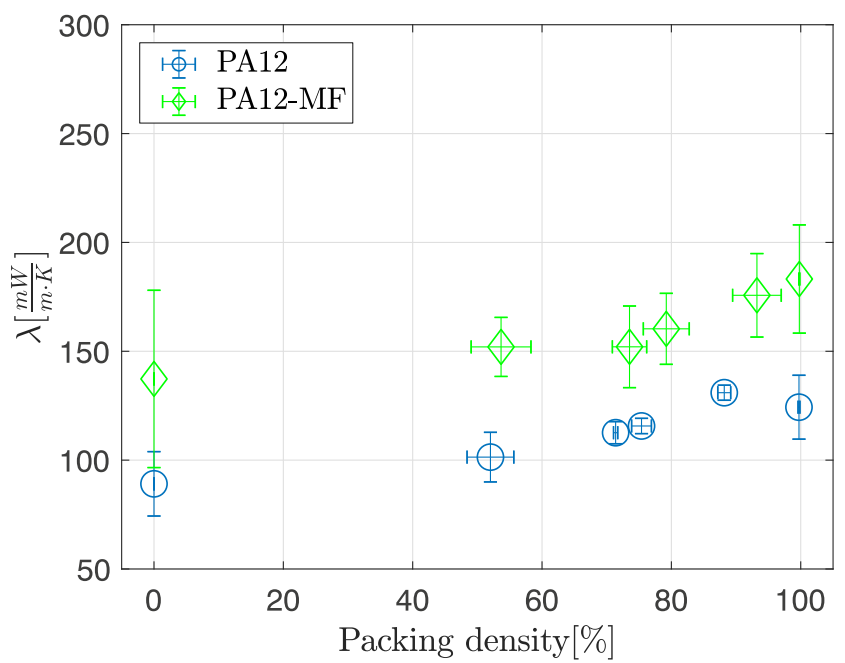

Fig. 10 Thermal conductivity vs. normalized packing density for PA12 and its composite PA12-MF, which contains Wollastonite fibers

thermal conductivity depending on the alignment. Since the fibers used in this study are characterized by a relative short length, roughly comparable with the PSD of the base polymer, an average value of $6000 \mathrm{mWm}^{-1} \mathrm{~K}^{-1}$ can be taken [22].

Again, the presence of the filler leads to an increase of thermal conductivity of about 50 to $150 \mathrm{mWm}^{-1} \mathrm{~K}^{-1}$, but not matching the expected value from the application of the rule of mixture (about $500 \mathrm{mWm}^{-1} \mathrm{~K}^{-1}$ ). The highest average value of $270 \mathrm{mWm}^{-1} \mathrm{~K}^{-1}$ for iCoPP-CF is reached at about $40 \%$ of the packing density and at 10 taps, and the large standard deviation can be explained possibly due to a favorable alignment of the short carbon fibers, which allowed to reach the very high value of $350 \mathrm{mWm}^{-1} \mathrm{~K}^{-1}$ in one of the three measurements (Fig. 11).

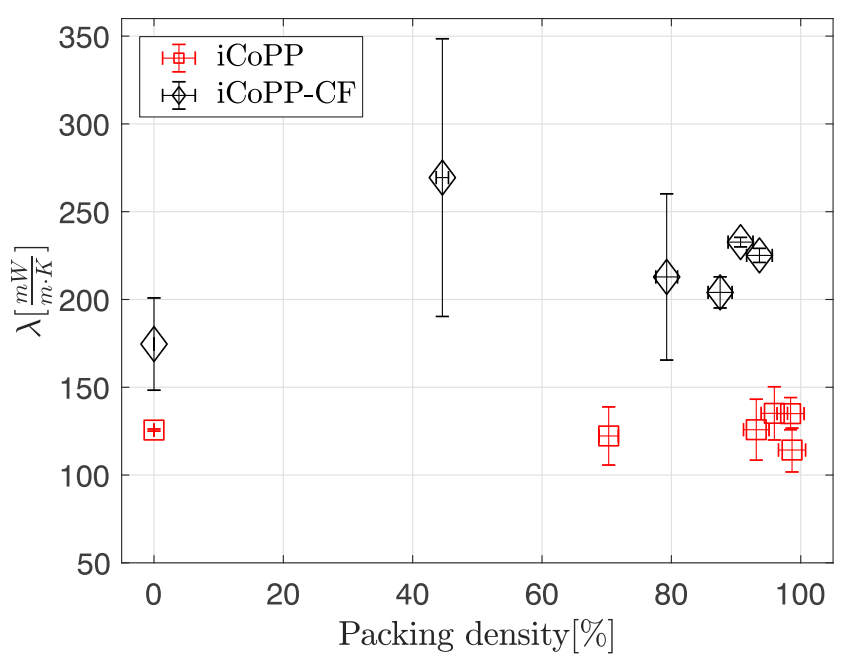

Fig. 11 Thermal conductivity vs. normalized packing density for iCoPP and its composite

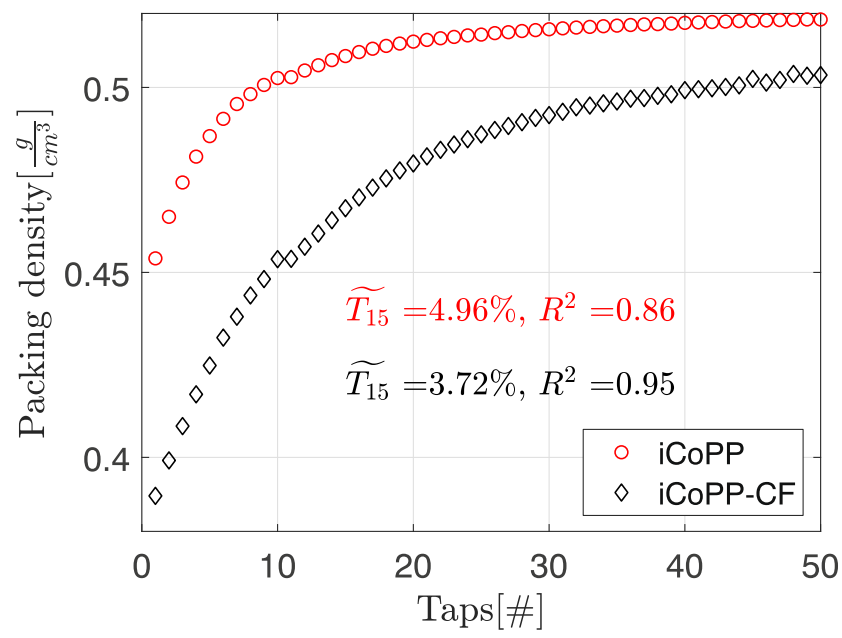

Fig. 12 Compaction curve for iCoPP and its composite with carbon fibers iCoPP-CF, first 50 taps

\subsection{Influence of fillers on flowability}

In general, the presence of fillers leads to a decrease of flowability since the rheological behavior of the powder is negatively affected by the presence of particles with elongated or acicular shapes. This can be observed also in this case, when looking at how the compaction curve for the first 50 taps changes upon addition of the filler, as shown in Fig. 12.

Even if the addition of carbon fibers should increase the absolute packing density of the powder, the flowability decrease is so detrimental that the actual bulk density is much lower than the one of neat iCoPP. Nevertheless, about the same value of tap density is reached by both powders, as confirmed by the sharp increase of the Hausner ratio, i.e., the ratio between tap and bulk density of the powder, from 1.15 (good) to 1.33 (passable).

The tapping modulus $\widetilde{T_{15}}$, obtained through a linear regression over the first 15 taps [10], is used to quantify the speed of compaction in normalized units. Since $\widetilde{T_{15}}$ is higher for iCoPP, iCoPP-CF behaves less well in terms of compaction flow.

\section{Conclusions and outlook}

A more precise knowledge on the influence of compaction and presence of fillers on thermal conductivity of polymer powders is of fundamental importance for building more precise thermal models of the PBF process, and in general to improve the process robustness in industrial applications.

In this work, an innovative setup to measure thermal conductivity of electrically non-conductive powders as a function of the packing density was developed and 
tested. The influence of packing density on the thermal conductivity was studied for four PBF-LB/P materials, two neat polymers and two blends. In general, for powders exhibiting a sufficiently large difference between bulk and tap density such as PA12, a positive linear trend could be observed between packing density and thermal conductivity, with an increase of thermal conductivity of about $40 \%$ to $125 \mathrm{mWm}^{-1} \mathrm{~K}^{-1}$. Better flowing powders, such as iCoPP, only presented a modest increase of about $10 \%$ to 135 $\mathrm{mWm}^{-1} \mathrm{~K}^{-1}$, since the difference between the bulk and tap density, which can be quantified using Hausner ratio, is very low.

The presence of fillers also influences the thermal conductivity of the base polymer, leading to an increase between 40 and $70 \%$ to a maximum of $225 \mathrm{mWm}^{-1} \mathrm{~K}^{-1}$ with carbon fibers. A significant drop in flowability has been observed upon addition of the filler, which could cause problems in the recoatability of the powder during the process. For this reason, the processing parameters would need to be changed in order to account for the completely different thermal behavior caused by the presence of the reinforcement material.

A continuation of the present work would be to improve the measuring setup with a more reliable current source and measure other AM relevant materials, an important step in order to better understand the complex thermal phenomena that regulate powder bed fusion of polymers.

Author contribution Francesco Sillani: conceptualization, methodology, investigation, software, formal analysis, writing-original draft, writing-review and editing, visualization. Fabian de Gasparo: investigation, software. Manfred Schmid: writing-review and editing, supervision. Konrad Wegener: writing-review and editing, supervision.

Funding Open access funding provided by Swiss Federal Institute of Technology Zurich.

\section{Declarations}

Conflict of interest All the authors certify that they have no affiliations with or involvement in any organization or entity with any financial interest or non-financial interest in the subject matter or materials discussed in this manuscript. The authors give their consent for publication.

Open Access This article is licensed under a Creative Commons Attribution 4.0 International License, which permits use, sharing, adaptation, distribution and reproduction in any medium or format, as long as you give appropriate credit to the original author(s) and the source, provide a link to the Creative Commons licence, and indicate if changes were made. The images or other third party material in this article are included in the article's Creative Commons licence, unless indicated otherwise in a credit line to the material. If material is not included in the article's Creative Commons licence and your intended use is not permitted by statutory regulation or exceeds the permitted use, you will need to obtain permission directly from the copyright holder. To view a copy of this licence, visit http://creativecommons. org/licenses/by/4.0/.

\section{References}

1. Wohlers TT, Campbell I (2017) Wohlers Report 2017 Technical report

2. ASTM International and ISO (2018) ISO/ASTM 52900 - 18: Additive manufacturing terminology

3. Schmid M (2018) Laser Sintering with Plastics. Hanser Publishers, Munich. ISBN 978-1-56990-683-5. https://doi.org/10.3139/9 781569906842.fm

4. Amado F, Schmid M, Wegener K (2011) Advances in SLS powder characterization. In: Proceedings of the international conference on solid freeform fabrication 2011 (SFF' 11). University of Texas, Austin, pp 438-452

5. Ziegelmeier S, Wollecke F, Tuck C, Goodridge R, Hague R (2013) Characterizing the bulk \& flow behaviour of LS polymer powders. In: Proceedings of the international conference on solid freeform fabrication 2013 (SFF' 13), pp 354-367

6. Vock S, Klöden B, Kirchner A, Weißgärber T, Kieback B (2019) Powders for powder bed fusion: a review. Progress in Additive Manufacturing, 2. ISSN 2363-9512. https://doi.org/10.1007/s40964-019-00078-6

7. Gotoh K, Masuda H, Higashitani K (2001) Powder technology handbook, vol 10. CRC Press, Boca Raton, p 1. ISBN 9780429190186. https://doi.org/10.1016/0921-5093(93)90508-c. https://www.taylorfrancis.com/books/9781439831885

8. ASTM International (2018) ASTM B417 - 18: Standard Test Method for Apparent Density of Non-Free-Flowing Metal Powders Using the Carney Funnel

9. ASTM International (2015) ASTM B527 - 15: Standard Test Method for Tap Density of Metal Powders and Compounds

10. Sillani F, Wagner D, Spurek M, Haferkamp L, Spierings A, Schmid M, Wegener K (2020) Compaction behavior of powder bed fusion feedstocks for metal and polymer additive manufacturing. Manuscript submitted for publication

11. Yuan M, Diller TT, Bourell D, Beaman J (2013) Thermal conductivity of polyamide 12 powder for use in laser sintering. Rapid Prototyp J 19(6):437-445, 9. ISSN 13552546. https://doi.org/10.1108/RPJ-11-2011-0123

12. Assael MJ, Antoniadis KD, Wakeham WA (2010) Historical evolution of the transient hot-wire technique. Int J Thermophys 31(6):1051-1072, 6. ISSN 0195928X. https://doi.org/10.1007/s10765-010-0814-9

13. De Groot JJ, Kestin J, Sookiazian H (1974) Instrument to measure the thermal conductivity of gases. Physica 75(3):454-482, 8. ISSN 00318914. https://doi.org/10.1016/0031-8914(74)90341-3

14. Healy JJ, de Groot JJ, Kestin J (1976) The theory of the transient hot-wire method for measuring thermal conductivity. Physica B+C 82(2):392-408, 4. ISSN 03784363. https://doi.org/10.1016/0378-4363(76)90203-5

15. Wei LC, Ehrlich LE, Powell-Palm MJ, Montgomery C, Beuth J, Malen JA (2018) Thermal conductivity of metal powders for powder bed additive manufacturing. Addit Manufact 21:201-208, 5. ISSN 22148604. https://doi.org/10.1016/j.addma.2018.02.002. https://linkinghub.elsevier.com/retrieve/pii/S2214860417306231

16. Giancoli DC (2004) Physics: Principles with Applications, vol 1. ISBN 0130606200 
17. Mark JE (ed) (2007) Physical Properties of Polymers Handbook. Springer, New York. ISBN 978-0-387-31235-4. https://doi.org/10.1007/978-0-387-69002-5

18. Schmid M, Amado F, Levy G (2011) icoPP - A new polyolefin for additive manufacturing (SLS). International Conference on Additive Manufacturing AM

19. Sillani F, Kleijnen RG, Vetterli M, Schmid M, Wegener K (2019) Selective laser sintering and multi jet fusion: Processinduced modification of the raw materials and analyses of parts performance. Additive Manufact 27:32-41, 5. ISSN 22148604. https://doi.org/10.1016/j.addma.2019.02.004
20. 3D Systems (2020) Datasheet Duraform HST. https://bit.ly/ 3pZpUVZ

21. Imerys (2017) Physical properties of wollastonite. https://bit.ly/ 2JbUmva

22. Bard S, Schönl F, Demleitner M, Altstädt V (2019) Influence of fiber volume content on thermal conductivity in transverse and fiber direction of carbon fiber-reinforced epoxy laminates. Materials, 12(7). ISSN 19961944. https://doi.org/10.3390/ma12071084

Publisher's note Springer Nature remains neutral with regard to jurisdictional claims in published maps and institutional affiliations. 